\title{
Predicting Activation Patterns in Cardiac Resynchronization Therapy Patients
}

\author{
Angela W C Lee ${ }^{1}$, Uyen C Nguyen ${ }^{2}$, Justin Gould ${ }^{1}$, Baldeep Sidhu ${ }^{1}$, Benjamin Sieniewicz ${ }^{1}$, Caroline \\ Mendonca Costa ${ }^{1}$, Fritz Prinzen ${ }^{2}$, Gernot Plank ${ }^{3}$, Christopher A Rinaldi ${ }^{1}$, Kevin Vernooy ${ }^{2}$, Steven A \\ Niederer ${ }^{1}$ \\ ${ }^{1}$ Kings' College London, London, United Kingdom \\ ${ }^{2}$ Maastricht University Medical Center (MUMC+), Cardiovascular Research Institute Maastricht \\ (CARIM), Maastricht, the Netherlands \\ ${ }^{3}$ Medical University of Graz, Graz, Austria
}

\begin{abstract}
Cardiac resynchronization therapy (CRT) is an effective treatment for heart failure patients. Suboptimal pacing timings and locations have been identified as causes for nonresponse to CRT. Patient specific computer models allow for the prediction of the electrical activation pattern on the ventricles, which can then be used to optimise CRT lead location.

The electrical activation of the heart depends on the underlying cardiac substrate. The electrical properties of the heart have been found to be heterogeneous, with scar, functional block, septal slowing, and fast endocardial conduction impacting the electrical activation across the ventricles.

Non-invasive data from 14 patients were used to create computer models of the heart. The patient specific models were then used to assess the importance of the heterogeneous cardiac substrates on accurately predicting the electrical activation pattern of the ventricles. Fast endocardial conduction was found to be the most important factor in accurately predicting the electrical activation of the heart in CRT patients.
\end{abstract}

\section{Introduction}

Cardiac resynchronization therapy (CRT) is used to treat heart failure patients with ventricular dyssynchrony. Suboptimal pacing timings and locations have been identified as causes for nonresponse to CRT [1]. Cardiac computer models can be used to simulate the electrical activation and thus optimize CRT $[2,3]$.

Experimental studies have found that the electrical properties of the heart varies heterogeneously throughout the ventricles $[4,5]$. The septum, functional block regions in the anterior or posterior regions of the left ventricle, and scarred tissues have been found to be associated with reductions in the conduction velocities [6-8]. While increased conduction velocities were found in the endocardial layer in the ventricles (fast endocardial conduction) [4].

The aim of this study was to identify the electrical properties of the human heart which are important for predicting activation patterns in CRT patients.

\section{Methods}

Patient-specific models were used to predict the electrical activation of the heart, with the conduction velocity fitted to the QRS duration for 14 patients. Delayed enhancement cardiac MRI was used to personalize the geometry and delineation of scar [9]. Intra-procedural coronary venous electro-anatomical mapping was performed during right ventricular pacing and the clinical measurements were used to identify the relative importance of scar, anterior or posterior functional block, slow septal conduction and fast endocardial conduction. Clinical measurements of the local activation times (LAT) were compared against the simulations to calculate the temporal errors. The distance errors were calculated as the product of the LAT error and the conduction velocity

\section{Results}

One-way ANOVA was used to compare the temporal and distance errors for the models incorporating the scar, functional block, slow septal conduction, and fast endocardial conduction. Differences were found in the distance errors for the models $(F(5,78)=8.87$, p-value $<0.0001$ ), though not for the temporal errors ( $\mathrm{p}$-value $>0.1$ ). Tukey post-hoc tests indicated that the fast endocardial conduction model had a significantly reduced mean distance error $(6.8 \mathrm{~mm})$ in comparison to the other models $(15.8-17.8 \mathrm{~mm})$.

\section{Conclusion}

In CRT patients the electrical activation pattern is dependent on the presence of fast conducting endocardial tissue, which may be important in predicting optimal pacing locations and timings. 


\section{References}

[1] Mullens, W., Grimm, R.a., Verga, T., Dresing, T., Starling, R.C., Wilkoff, B.L., and Tang, W.H.W.: 'Insights From a Cardiac Resynchronization Optimization Clinic as Part of a Heart Failure Disease Management Program', Journal of the American College of Cardiology, 2009, 53, (9), pp. 765-773

[2] Lee, A.W., Crozier, A., Hyde, E.R., Lamata, P., Truong, M., Sohal, M., Jackson, T., Behar, J.M., Claridge, S., Shetty, A., Sammut, E., Plank, G., Rinaldi, C.A., and Niederer, S.: 'Biophysical Modeling to Determine the Optimization of Left Ventricular Pacing Site and AV/VV Delays in the Acute and Chronic Phase of Cardiac Resynchronization Therapy', J Cardiovasc Electrophysiol, 2017, 28, (2), pp. 208-215

[3] Villongco, C.T., Krummen, D.E., Omens, J.H., and McCulloch, A.D.: 'Non-invasive, model-based measures of ventricular electrical dyssynchrony for predicting CRT outcomes', Europace, 2016, 18, (suppl 4), pp. iv104-iv112

[4] Draper, M., and Mya-Tu, M.: 'A comparison of the conduction velocity in cardiac tissues of various mammals', Experimental Physiology, 1959, 44, (1), pp. 91-109

[5] Strik, M., Ploux, S., Vernooy, K., and Prinzen, F.W.: 'Cardiac resynchronization therapy: refocus on the electrical substrate', Circulation journal : official journal of the Japanese Circulation Society, 2011, 75, (6), pp. 1297-1304

[6] de Bakker, J.M., van Capelle, F.J., Janse, M.J., Tasseron, S., Vermeulen, J.T., de Jonge, N., and Lahpor, J.R.: 'Slow conduction in the infarcted human heart. 'Zigzag' course of activation', Circulation, 1993, 88, (3), pp. 915-926
Hemodynamic Effect of Left Ventricular and Biventricular Endocardial PacingCLINICAL PERSPECTIVE: Insights From Electroanatomical Mapping', Circulation: Arrhythmia and Electrophysiology, 2014, 7, (2), pp. 251-258

[8] Auricchio, A., Fantoni, C., Regoli, F., Carbucicchio, C., Goette, A., Geller, C., Kloss, M., and Klein, H.: 'Characterization of Left Ventricular Activation in Patients with Heart Failure and Left Bundle-Branch Block', Circulation, 2004, 109, (9), pp. 1133-1139

[9] Nguyen, U.C., Mafi-Rad, M., Aben, J.P., Smulders, M.W., Engels, E.B., van Stipdonk, A.M., Luermans, J.G., Bekkers, S.C., Prinzen, F.W., and Vernooy, K.: 'A novel approach for left ventricular lead placement in cardiac resynchronization therapy: Intraprocedural integration of coronary venous electroanatomic mapping with delayed enhancement cardiac magnetic resonance imaging', Heart Rhythm, 2017, 14, (1), pp. 110-119

Address for correspondence:

Angela Lee Imaging Sciences \& Biomedical Engineering Division of Imaging Sciences \& Biomedical Engineering 4th Floor, Lambeth Wing

St. Thomas' Hospital

Westminster Bridge Road

London SE1 7EH

angela.lee@kcl.ac.uk

[7] Sohal, M., Shetty, A., Niederer, S., Chen, Z., Jackson, T., Sammut, E., Bostock, J., Razavi, R., Prinzen, F., and Rinaldi, C.A.: 'Delayed Trans-Septal Activation Results in Comparable 